\title{
Right heart thrombi in pulmonary embolism
}

\author{
Deisy Barrios ${ }^{1,8}$, Vladimir Rosa-Salazar ${ }^{2,8}$, David Jiménez ${ }^{1}$, Raquel Morillo ${ }^{1}$, \\ Alfonso Muriel ${ }^{1}$, Jorge del Toro ${ }^{3}$, Luciano López-Jiménez ${ }^{4}$, \\ Dominique Farge-Bancel ${ }^{5}$, Roger Yusen ${ }^{6}$ and Manuel Monreal ${ }^{7}$, for the RIETE \\ investigators ${ }^{9}$
}

Affiliations: ${ }^{1}$ Respiratory Dept, Ramón y Cajal Hospital and Universidad de Alcala (IRYCIS), Madrid, Spain. ${ }^{2}$ Dept of Internal Medicine, Hospital Clinico Universitario Virgen de la Arrixaca, Murcia, Spain. ${ }^{3}$ Dept of Internal Medicine, Hospital General Universitario Gregorio Marañón, Madrid, Spain. ${ }^{4}$ Dept of Internal Medicine, Hospital Universitario Reina Sofía, Córdoba, Spain. ${ }^{5}$ Dept of Internal Medicine and Pathology, Hôpital Saint-Louis, Paris, France. ${ }^{6}$ Divisions of Pulmonary and Critical Care Medicine and General Medical Sciences, Washington University School of Medicine, St. Louis, MO, USA. ${ }^{7}$ Dept of Internal Medicine, Hospital Universitari Germans Trias i Pujol, Badalona, Universidad Católica de Murcia, Spain. ${ }^{8}$ Both authors contributed equally to the manuscript. ${ }^{9} \mathrm{~A}$ full list of the RIETE investigators is given in the acknowledgements section.

Correspondence: David Jiménez, Respiratory Dept and Medicine Dept, Ramón y Cajal Hospital and Universidad de Alcala (IRYCIS), 28034 Madrid, Spain. E-mail: djimenez.hrclagmail.com

ABSTRACT There is a lack of comprehensive data on the prevalence, predictors and prognostic significance of right heart thrombi (RHT) in pulmonary embolism.

In this study of patients with pulmonary embolism from the Registro Informatizado de la Enfermedad TromboEmbólica (RIETE) registry, we assessed the prevalence and predictors of RHT, and the association between the presence of RHT and the outcomes of all-cause mortality, pulmonary embolism-related mortality, recurrences, and major bleeding through 30 days after initiation of pulmonary embolism treatment.

Of 12441 patients with pulmonary embolism and baseline echocardiographic data, $2.6 \%$ had RHT. The following increased the risk of RHT: younger age, previous bleeding, congestive heart failure, cancer, syncope, systolic blood pressure $<100 \mathrm{mmHg}$, and arterial oxyhaemoglobin saturation $<90 \%$. Patients with RHT were significantly more likely to die from any cause (adjusted OR 2.50 (95\% CI 1.62-3.84); p<0.001) and from pulmonary embolism (adjusted OR 4.29 (95\% CI 2.45-7.48); p<0.001) during follow-up. RHT was associated with an increased risk of recurrence during follow-up ( $1.8 \%$ versus $0.7 \%$; $\mathrm{p}=0.04)$. Major bleeding was similar in patients with and without RHT.

In patients presenting with pulmonary embolism, RHT is relatively infrequent. Patients with RHT had a worse outcome when compared with those without RHT.

@ERSpublications

Right heart thrombus is an independent predictor of death in patients with acute pulmonary embolism http://ow.ly/y5i5302CDCS 


\section{Introduction}

Pulmonary embolism is a potentially life-threatening cardiovascular condition [1]. Acute pulmonary embolism has a wide spectrum of clinical syndromes and varying clinical outcomes [2,3]. Assessment of prognosis is of crucial importance for the therapeutic choices in patients with pulmonary embolism $[4,5]$.

It is commonly believed that right heart thrombi (RHT) are rare in patients with acute symptomatic pulmonary embolism $[6,7]$. In these patients, the increased use of two-dimensional echocardiography for prognostic purposes has led to increased detection of RHT, and the true frequency of this complication is not well established. There is also limited documentation concerning the prognostic significance of concomitant RHT at the time of acute pulmonary embolism diagnosis [8]. Therapeutic options in these patients consist of anticoagulation, thrombolysis or surgical embolectomy, but the optimal management remains controversial.

The Registro Informatizado de la Enfermedad TromboEmbólica (RIETE) registry is an ongoing, multicentre, international, prospective registry of consecutive patients with symptomatic, objectively confirmed, acute venous thromboembolism (VTE) [9-11]. RIETE has sufficiently large sample size and study duration to compare the clinical characteristics and outcome of patients with RHT and of similarly treated patients without RHT. The objective of this study was thus three-fold: to estimate the prevalence of RHT; to identify the strongest predictors of RHT in pulmonary embolism patients; and to assess the association between RHT and short-term clinical outcomes.

\section{Methods}

\section{Study design}

This retrospective study used prospectively collected data from patients enrolled in the RIETE registry $[1,9]$. All patients provided written or oral consent for participation in the registry in accordance with local ethics committee requirements.

\section{Patients}

At each participating site, RIETE investigators aimed to enroll consecutive patients that had acute symptomatic or asymptomatic VTE confirmed by objective testing that consisted of high probability ventilation-perfusion scintigraphy [12], positive contrast-enhanced, pulmonary embolism-protocol, helical chest computed tomography for pulmonary embolism [13], or lower limb venous compression ultrasonography positive for proximal deep vein thrombosis (DVT) [14]. Only patients diagnosed with acute symptomatic pulmonary embolism were eligible. The only exclusion criterion consisted of inability to complete transthoracic echocardiography testing.

\section{Baseline variables}

Patients enrolled in RIETE had data collected from around the time of pulmonary embolism diagnosis that included but was not limited to: age; sex; body weight; presence of coexisting conditions such as chronic heart or lung disease; recent ( $<30$ days prior to pulmonary embolism) major bleeding; presence of risk factors for pulmonary embolism including active cancer (defined as newly diagnosed cancer or cancer undergoing treatment (i.e. surgery, chemotherapy, radiotherapy, hormonal or support therapy), recent immobility (defined as non-surgical patients assigned to bed rest with bathroom privileges for $\geqslant 4$ days in the 2 months prior to pulmonary embolism diagnosis), surgery (defined as those who had undergone major surgery in the 2 months prior to pulmonary embolism); clinical signs and symptoms on admission, including heart rate and systolic blood pressure; and laboratory results at hospital admission that included haemoglobin, platelet count and serum creatinine.

\section{Study outcomes}

This study used all-cause mortality through 30 days following the diagnosis of pulmonary embolism as the primary endpoint, and 30-day pulmonary embolism-related mortality, recurrent VTE, and major bleeding as secondary endpoints. The RIETE investigators used medical record review to assess vital status. For patients that died, further medical record review, and proxy interviews when necessary, assisted with determining date and cause of death. For deaths confirmed by autopsy or those following a clinically severe pulmonary embolism, either initially or shortly after an objectively confirmed recurrent event, in the absence of any alternative diagnosis, the investigators were instructed to judge death as due to fatal pulmonary embolism. Clinicians at RIETE enrolling sites managed patients with suspected recurrences according to their local practice. Typically, the RIETE investigators defined recurrent DVT as a new noncompressible vein segment, or an increase of the vein diameter by at least $4 \mathrm{~mm}$ compared with the last available measurement on venous ultrasonography [15]; recurrent pulmonary embolism as a new ventilation-perfusion mismatch on lung scan or a new intraluminal filling defect on spiral computed tomography of the chest [13]; and major bleeding episodes as those that required a transfusion of at least 2 units of blood, were retroperitoneal, spinal or intracranial, or were fatal [16]. 
Treatment and follow-up

Clinicians at RIETE-enrolling sites managed patients according to their local practice (i.e. no standardisation of treatment). In the RIETE registry, most patients received initial anticoagulation with intravenous unfractionated heparin or subcutaneous low-molecular-weight heparin or fondaparinux, and overlap and long-term therapy with an oral anticoagulant. Clinicians administered thrombolytic treatment and/or inotropic support as deemed appropriate. In general, clinicians used thrombolytic treatment of acute pulmonary embolism in patients with cardiogenic shock (e.g. persistent systolic arterial pressure $<90 \mathrm{mmHg}$ in the setting of clinical signs of organ hypoperfusion (clouded sensorium, oliguria, cold and clammy skin or lactic acidosis)). In most patients with a contraindication to or a failure of anticoagulant treatment (i.e. VTE recurrence while on therapeutic doses of anticoagulation), clinicians used inferior vena cava filter therapy followed by anticoagulation if the contraindication resolved. RIETE recorded information related to patient outcomes through 3 months after the diagnosis of the acute pulmonary embolism, and this study analysed outcomes through 30 days after initiation of pulmonary embolism treatment.

\section{Statistical analysis}

We used Chi-squared or Fisher's exact tests to compare categorical data between groups. We used the Kolmogorov-Smirnov test to assess continuous data for a normal distribution. We used two-tailed unpaired t-tests to compare normally distributed continuous data between two groups, and we used the Mann-Whitney U-test for non-normally distributed continuous data comparisons.

We evaluated potential demographic and clinical risk factors for RHT in the entire cohort with the use of univariable logistic regression analysis. All variables with a univariable level of significance of $<0.1$ were included in a backward, stepwise multivariable logistic regression model.

To estimate the outcomes of time to death, Kaplan-Meier probabilities were computed [17], and differences between the groups were assessed with the log-rank test. Survival analyses censored for loss to follow-up (none) and end of study. The study used logistic regression to assess for an independent association between the presence of RHT at the time of presentation with pulmonary embolism and the outcome measures. For the manual backward stepwise multivariable logistic regression model, we assessed variables that had a significance level of $\mathrm{p}<0.1$ in univariable analyses. The model retained those variables associated with the outcome at a significance level of $\mathrm{p}<0.05$. Interaction terms with type of treatment (anticoagulation versus thrombolysis or surgery) and severity of pulmonary embolism (low-, intermediate- and high-risk pulmonary embolism, according to the European Society of Cardiology prognostic model) [5] were tested for significance at an $\alpha$-level of 0.05 . To test the robustness of the findings, the effects of excluding haemodynamically unstable patients (i.e. systolic blood pressure $<90 \mathrm{mmHg}$ ), were assessed. Analyses were performed using SPSS, version 20 for the PC (SPSS, Inc. Chicago, IL, USA).

\section{Results}

We identified 30254 patients with objectively confirmed symptomatic pulmonary embolism enrolled in RIETE during the time period of January 1, 2001, through December 15, 2015. The study excluded 17813 patients because they did not have complete baseline echocardiographic data required for analyses $(58.9 \%$; 95\% CI 58.3-59.4\%). Patients who were ineligible were older and had more comorbid diseases (cancer, recent surgery, immobility and recent bleeding), but fewer signs of clinical severity (syncope, tachycardia and hypotension) compared with those that were eligible. A total of 12441 patients (6718 women and 5723 men) were included in the present study (figure 1).

Overall, 325 patients (2.6\%; 95\% CI 2.3-2.9\%) had RHT and 12116 did not. Patients with RHT differed significantly from those without RHT in pre-existing medical conditions, and in relevant clinical, physiological and laboratory parameters. As shown in table 1, patients with RHT had a higher prevalence of chronic heart disease, cancer, immobilisation or recent major bleeding compared with those without RHT. Patients with RHT were more haemodynamically compromised than patients without RHT, with lower systolic blood pressure, higher heart rate and more frequent hypoxaemia and syncope (table 1). Patients with RHT also had a higher prevalence of renal insufficiency. With respect to similarities, age, prevalence of male sex, dyspnoea, chest pain and concomitant DVT were almost identical in patients with and without RHT, as was the frequency of chronic obstructive pulmonary disease, history of VTE and recent surgery. In the multivariable model, younger age (adjusted OR 1.01 per year; 95\% CI 1.01-1.02), previous bleeding (adjusted OR 2.56; 95\% CI 1.51-4.34), congestive heart failure (adjusted OR 2.06; 95\% CI 1.51-2.81), cancer (adjusted OR 1.46; 95\% CI 1.11-1.91), syncope (adjusted OR 1.83; 95\% CI 1.42-2.36), systolic blood pressure $<100 \mathrm{mmHg}$ (adjusted OR 1.97; 95\% CI 1.48-2.63) and arterial oxyhaemoglobin saturation $<90 \%$ (adjusted OR 1.58; 95\% CI 1.23-2.03) remained significantly associated with the presence of RHT. Treatment was significantly different between the two groups: $17.8 \%$ of patients with RHT received thrombolysis, compared with $4.9 \%$ of patients without RHT and $5.3 \%$ in the overall 
30254 patients with acute symptomatic $\mathrm{PE}$ were enrolled in RIETE

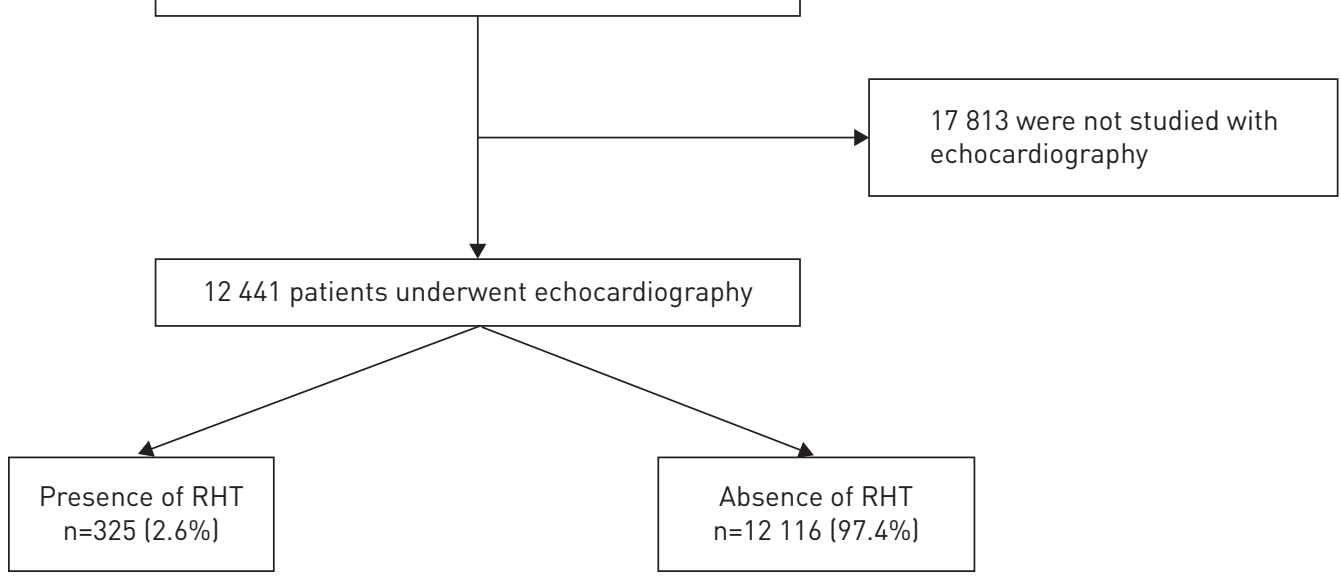

FIGURE 1 Patient flow diagram. PE: pulmonary embolism; RIETE: Registro Informatizado de la Enfermedad TromboEmbólica; RHT: right heart thrombi.

RIETE cohort. Embolectomy was performed in $3.7 \%$ of patients with RHT and in $0.9 \%$ of patients without RHT (table 1).

\section{RHT and right ventricular function}

Patients with RHT had double the frequency of right ventricular hypokinesis ( $54.3 \%$ versus $23.4 \%$; $<<0.001)$ and more often had elevated cardiac troponin $(51.8 \%$ versus $36.3 \% ; \mathrm{p}<0.001)$ and BNP levels $(87.5 \%$ versus 70.5\%; p $<0.01)$. Patients with RHT had significantly higher systolic pulmonary artery pressure $(54.5 \pm 19.3 \mathrm{mmHg})$ than those without RHT $(45.1 \pm 17.3 \mathrm{mmHg})(\mathrm{p}<0.001)$. Tricuspid annular plane systolic excursion $(17.5 \pm 5.8 \mathrm{~mm}$ versus $20.1 \pm 6.0 \mathrm{~mm}$; $\mathrm{p}<0.001)$ was significantly lower among patients with RHT.

\section{Outcome}

Outcome data were available for all patients through the 30-day study follow-up (table 2). Overall, 382 (3.1\%) out of 12441 patients died (95\% CI 2.8-3.4\%). 122 (1.0\%) out of 12441 patients (95\% CI 0.8-1.2\%) died from pulmonary embolism, and 260 (2.1\%) out of 12441 patients (95\% CI 1.8-2.4\%) died from other causes. Other causes of death consisted of cancer (64 (0.5\%) out of 12441 patients), respiratory failure (42 $(0.3 \%)$ of 12441 patients), infection (24 (0.2\%) out of 12441 patients), major bleeding (22 (0.2\%) out of 12441 patients), congestive heart failure (18 (0.1\%) out of 12441 patients), miscellaneous diseases (58 ( $0.5 \%)$ out of 12441 patients) and unknown (32 (0.3\%) out of 12441 patients). 28 deaths (8.6\%) out of 325 patients (95\% CI 5.8-12.2\%) occurred in the group of patients entering the study with acute pulmonary embolism and RHT, whereas 354 deaths (2.9\%) out of 12116 patients (95\% CI 2.6-3.2\%) occurred in the group without RHT (absolute difference 5.7\%, 95\% CI 3.1-9.3\%; $\mathrm{p}<0.001$ ). Patients with acute pulmonary embolism and RHT had a significantly higher cumulative mortality than the patients with acute pulmonary embolism without RHT $(\mathrm{p}<0.001, \log$ rank test) (figure 2$)$. The multivariable model found that age (adjusted OR 1.03, 95\% CI 1.02-1.04; $\mathrm{p}<0.001$ ), cancer (adjusted OR 4.03, 95\% CI 3.26-4.98; $\mathrm{p}<0.001$ ), recent major bleeding (adjusted OR 1.73, 95\% CI 1.03-2.88; $\mathrm{p}=0.04$ ), recent immobility (adjusted OR 2.34, 95\% CI 1.87-2.92; p<0.001), systolic blood pressure <100 mmHg (adjusted OR 2.24, 95\% CI 1.71-2.93; $\mathrm{p}<0.001$ ), heart rate $\geqslant 110$ beats. $\mathrm{min}^{-1}$ (adjusted OR 1.28, 95\% CI 1.01-1.63; $\mathrm{p}=0.04$ ) and RHT (adjusted OR 2.50, 95\% CI 1.62-3.84; $\mathrm{p}<0.001$ ) independently predicted 30-day all-cause mortality (table 3 ).

Pulmonary embolism-related mortality within 30 days of pulmonary embolism diagnosis occurred more frequently in patients with RHT (5.2\% versus $0.9 \%$; absolute difference $4.3 \%, 95 \%$ CI of the absolute difference 2.4-7.4\%; $\mathrm{p}<0.001$ ). Recurrent VTE within 30 days of pulmonary embolism diagnosis occurred in six patients $(1.8 \%, 95 \%$ CI $0.7-4.0 \%)$ entering the study with RHT and in 87 patients $(0.7 \%, 95 \%$ CI $0.6-0.9 \%$ ) without RHT (absolute difference $1.1 \%$, 95\% CI of the absolute difference $0.1-3.2 \%$; $\mathrm{p}=0.04$ ) (table 2). 440 patients (3.5\%, 95\% CI 13.2-3.9\%) suffered a major bleeding episode. 15 bleeds (15 (4.6\%) out of 325 patients, 95\% CI 2.6-7.5\%) occurred in the group of patients entering the study with acute pulmonary embolism and RHT, whereas 425 bleeds (425 (3.5\%) out of 12116 patients, 95\% CI 3.2-3.8\%) occurred in the group without RHT (absolute difference 1.1\%, 95\% CI of the absolute difference $-0.7-4.0 \% ; \mathrm{p}=0.36$ ). Figure 3 shows a significant interaction between the type of treatment (anticoagulation versus thrombolysis or surgery), the severity of pulmonary embolism (low-, intermediate- and high-risk) 
TABLE 1 Clinical characteristics of patients with or without right heart thrombi

\begin{tabular}{|c|c|c|c|}
\hline & $\begin{array}{l}\text { Presence of right } \\
\text { heart thrombi }\end{array}$ & $\begin{array}{l}\text { Absence of right } \\
\text { heart thrombi }\end{array}$ & p-value \\
\hline Patients n & 325 & 12116 & \\
\hline \multicolumn{4}{|l|}{ Clinical characteristics } \\
\hline Age years & $64.2 \pm 17.3$ & $65.9 \pm 17.2$ & 0.08 \\
\hline Age $>80$ years & 57 (17.5) & 2485 (20.5) & 0.19 \\
\hline Male sex & $149(45.8)$ & $5574(46.0)$ & 0.95 \\
\hline Weight kg & $76.2 \pm 15.4$ & $77.2 \pm 16.5$ & 0.26 \\
\hline \multicolumn{4}{|l|}{ Risk factors for VTE } \\
\hline History of VTE & $54(16.6)$ & $1744(14.4)$ & 0.26 \\
\hline Cancer $^{\#}$ & 73 (22.5) & $2064(17.0)$ & 0.01 \\
\hline Recent surgery" & $38(11.7)$ & $1286(10.6)$ & 0.53 \\
\hline Immobilisation for $\geqslant 4$ days $^{+}$ & $81(24.9)$ & $2386(19.7)$ & 0.02 \\
\hline \multicolumn{4}{|l|}{ Comorbid diseases } \\
\hline Chronic lung disease & $54(16.6)$ & $1760(14.5)$ & 0.29 \\
\hline Chronic heart disease & 60 (18.5) & $1227(10.1)$ & $<0.001$ \\
\hline Recent major bleeding & $16(4.9)$ & 235 (1.9) & $<0.001$ \\
\hline \multicolumn{4}{|l|}{ Clinical symptoms and signs at presentation } \\
\hline Syncope & $98(30.1)$ & $2138(17.6)$ & $<0.001$ \\
\hline Chest pain & $153(47.1)$ & 5997 (49.5) & 0.45 \\
\hline Dyspnoea & 279 (85.8) & $10116(83.5)$ & 0.23 \\
\hline Heart rate $\geqslant 110$ beats $\cdot \mathrm{min}^{-1}$ & $108(33.2)$ & $2707(22.3)$ & $<0.001$ \\
\hline Arterial oxyhaemoglobin saturation $<90 \%$ & $103(31.7)$ & $2499(20.6)$ & $<0.001$ \\
\hline Systolic blood pressure $<100 \mathrm{mmHg}$ & $72(22.1)$ & $1145(3.5)$ & $<0.001$ \\
\hline Concomitant deep vein thrombosis & $151(46.5)$ & 5396 (44.5) & 0.49 \\
\hline \multicolumn{4}{|l|}{ Simplified PESI } \\
\hline Low risk & $93(28.6)$ & $4970(41.0)$ & $<0.001$ \\
\hline High risk & $232(71.4)$ & $7146(59.0)$ & $<0.001$ \\
\hline \multicolumn{4}{|l|}{ Echocardiography and cardiac biomarkers } \\
\hline RV hypokinesis ( $\mathrm{n}=10918)$ & $157 / 289(54.3)$ & $2492 / 10629(23.4)$ & $<0.001$ \\
\hline $\mathrm{RV} / \mathrm{LV}>1(\mathrm{n}=1671)$ & $6 / 40(15.0)$ & $322 / 1631(19.7)$ & 0.46 \\
\hline Systolic pulmonary pressure $\mathrm{mmHg}$ & $54.5 \pm 19.3$ & $45.1 \pm 17.3$ & $<0.001$ \\
\hline TAPSE $\mathrm{mm}$ & $17.5 \pm 5.8$ & $20.1 \pm 6.0$ & $<0.001$ \\
\hline Elevated troponin $(n=7419)$ & $114 / 220(51.8)$ & $2611 / 7199(36.3)$ & $<0.001$ \\
\hline BNP $>100 \mathrm{pg} \cdot \mathrm{mL}^{-1}(\mathrm{n}=1555)$ & $42 / 48(87.5)$ & $1063 / 1507(70.5)$ & $<0.01$ \\
\hline \multicolumn{4}{|l|}{ Laboratory findings } \\
\hline Abnormal creatinine levels (>2 $\mathrm{mg} \cdot \mathrm{dL}^{-1}$ ) & $84(25.8)$ & $2266(18.7)$ & $<0.001$ \\
\hline Haemoglobin $\mathrm{g} \cdot \mathrm{dL}^{-1}$ & $13.3 \pm 8.7$ & $13.2 \pm 3.3$ & 0.64 \\
\hline \multicolumn{4}{|l|}{ Treatment used } \\
\hline Unfractionated heparin & 125 (38.5) & $1714(14.1)$ & $<0.001$ \\
\hline Low-molecular-weight heparin & $212(65.2)$ & $10577(87.3)$ & $<0.001$ \\
\hline Thrombolysis & $58(17.8)$ & $597(4.9)$ & $<0.001$ \\
\hline Embolectomy & $12(3.7)$ & $104(0.9)$ & $<0.001$ \\
\hline Inferior vena cava filter & $15(4.6)$ & 396 (3.3) & 0.18 \\
\hline
\end{tabular}

Data are presented as mean \pm SD, $n(\%)$ or $n / N(\%)$, unless otherwise stated. VTE: venous thromboembolism; PESI: Pulmonary Embolism Severity Index; RV: right ventricle; LV: left ventricle; TAPSE: tricuspid annular plane systolic excursion; BNP: brain natriuretic peptide. " : active or under treatment in the last year; ${ }^{\text {I: }}$ in the previous month; ${ }^{+}$: immobilised patients defined as non-surgical patients who had been immobilised (i.e. total bed rest with bathroom privileges) for $\geqslant 4$ days in the month prior to pulmonary embolism diagnosis.

and the prognostic effect of RHT as a forest plot. RHT significantly predicted 30-day all-cause mortality among those patients who received anticoagulant therapy (OR 3.24, 95\% CI 2.05-5.13), but not among those patients who received thrombolysis or surgery (OR 0.92, 95\% CI $0.30-2.82$ ) ( $\mathrm{p}<0.01$ for interaction). Likewise, RHT significantly predicted 30-day all-cause mortality among low- (OR 6.71, 95\% CI 2.55-17.66) and intermediate-risk patients (OR 2.62, 95\% CI 1.56-4.40), but not among high-risk patients (OR 1.03, 95\% CI 0.36-2.99) ( $\mathrm{p}=0.02$ for interaction).

Using the same model variables, RHT remained an independent predictor of all-cause mortality when hypotensive patients were excluded from the analysis (adjusted OR 3.31, 95\% CI 2.09-5.24; p<0.001). After adjustment, RHT at the time of presentation was also independently significantly associated with 
TABLE 2 30-day clinical events after diagnosis and treatment for patients with acute symptomatic pulmonary embolism

\begin{tabular}{lcccc} 
& All patients & $\begin{array}{c}\text { Presence of right } \\
\text { heart thrombi }\end{array}$ & $\begin{array}{c}\text { Absence of right } \\
\text { heart thrombi }\end{array}$ & p-value \\
\hline $\begin{array}{l}\text { Patients n } \\
\text { Primary outcome }\end{array}$ & 12441 & 325 & 12116 & \\
$\quad \begin{array}{l}\text { All-cause death } \\
\quad \text { Pulmonary embolism-related death }\end{array}$ & $382(3.1)$ & $28(8.6)$ & $354(2.9)$ & $<0.001$ \\
$\begin{array}{l}\text { Secondary outcomes } \\
\quad \text { Recurrent pulmonary embolism }\end{array}$ & $122(1.0)$ & $17(5.2)$ & $105(0.9)$ & $<0.001$ \\
$\quad$ Major bleeding & $430(3.5)$ & $6(1.8)$ & $87(0.7 \%)$ & 0.04 \\
\hline
\end{tabular}

Data are presented as $\mathrm{n}(\%)$, unless otherwise stated.

pulmonary embolism-related death (adjusted OR 7.71, 95\% CI 4.34-13.69; p<0.001) and recurrent VTE within 30 days of pulmonary embolism diagnosis (adjusted OR 2.48, 95\% CI 1.06-5.82; p=0.04).

\section{Discussion}

Uncertainty surrounding the prevalence and prognostic significance of RHT exists. The results of our study show that RHT affect approximately $3 \%$ of patients with an episode of acute symptomatic pulmonary embolism. Also, patients with RHT had a worse outcome when compared with those without RHT.

The prevalence of RHT in the study cohort confirms the current belief that this condition is uncommon [18]. The only large-scale population-based study in this field published up to now reported a prevalence of echocardiographically detected RHT in patients with pulmonary embolism of 3.8\% [19]. For our prevalence estimates, we excluded patients who were not studied with echocardiography. Since potential risk factors for RHT (younger age, signs of clinical severity) were less frequent among patients who were ineligible compared to those that were eligible, we believe that our estimate of the prevalence of RHT should be viewed as the upper limit.

Prior evidence suggests that the risk of RHT is greatly increased among pulmonary embolism patients with heart failure and more haemodynamic compromise [19]. Heart failure showed a high predictive ability for RHT in our study. Though it has been postulated that only very large emboli arising from pelvic or proximal leg veins become lodged in the right heart [20], stasis in the dilated right heart due either to acute severe pulmonary embolism or pre-existing congestive heart failure, or both, seems to enhance the risk of RHT, regardless of whether it is due to in situ thrombosis or to entrapment of transiting thrombi. In our study, recent major bleeding was clearly associated with an increased risk of RHT. However, it should be noted that clinicians typically used suboptimal doses of anticoagulants in patients with previous episodes of major bleeding, contributing to the size of this increase in risk.

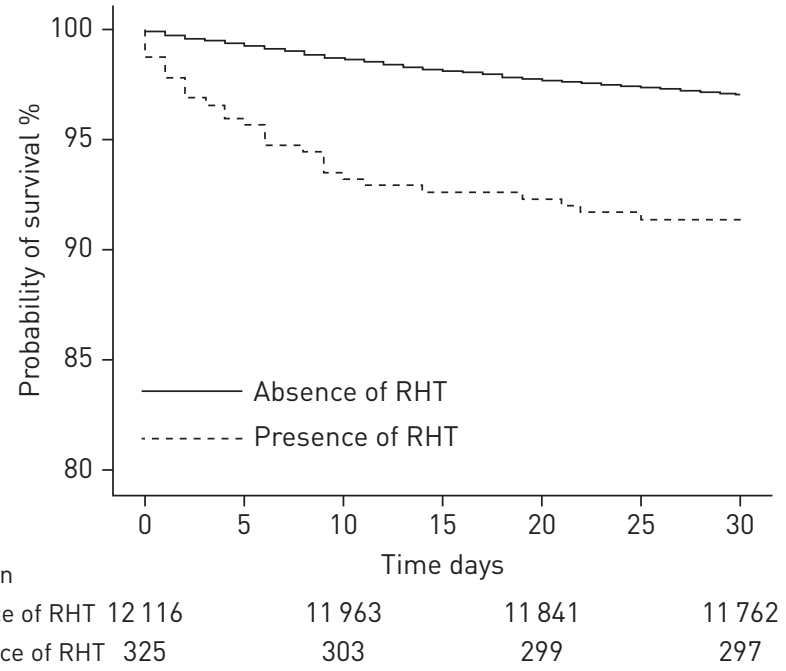

FIGURE 2 Kaplan-Meier estimates of survival (primary end-point, all-cause mortalityl in patients stratified by presence or absence of right heart thrombi (RHT). 


\begin{tabular}{|c|c|c|c|c|}
\hline Risk factor & $\begin{array}{l}\text { Unadjusted OR } \\
\text { (95\% CI) }\end{array}$ & p-value & $\begin{array}{c}\text { Adjusted OR } \\
\text { (95\% CI) }\end{array}$ & p-value \\
\hline Age per year & $1.03(1.02-1.04)$ & $<0.001$ & $1.03(1.02-1.04)$ & $<0.001$ \\
\hline Cancer $^{\#}$ & $4.03(3.26-4.99)$ & $<0.001$ & $4.03(3.26-4.98)$ & $<0.001$ \\
\hline Recent major bleeding & $1.82(1.09-3.04)$ & 0.02 & $1.73(1.03-2.88)$ & 0.04 \\
\hline Congestive heart failure & $1.28(0.96-1.70)$ & 0.09 & & \\
\hline Immobilisation" & $2.25(1.80-2.83)$ & $<0.001$ & $2.34(1.87-2.92)$ & $<0.001$ \\
\hline Syncope & $0.93(0.71-1.23)$ & 0.63 & & \\
\hline Systolic blood pressure $<100 \mathrm{mmHg}$ & $2.14(1.62-2.83)$ & $<0.001$ & $2.24(1.71-2.93)$ & $<0.001$ \\
\hline Heart rate $\geqslant 110$ beats $\cdot \min ^{-1}$ & $1.23(0.97-1.58)$ & 0.09 & $1.28(1.01-1.63)$ & 0.04 \\
\hline Arterial oxyhaemoglobin saturation $<90 \%$ & $1.20(0.94-1.52)$ & 0.14 & & \\
\hline Right heart thrombi & $2.46(1.60-3.80)$ & $<0.001$ & $2.50(1.62-3.84)$ & $<0.001$ \\
\hline
\end{tabular}

The Right Heart Thrombi European Registry was a prospective, international registry that reported the prognostic significance of RHT characteristics in patients with acute pulmonary embolism [8]. The main finding was that the clinical prognostic scores (i.e. simplified pulmonary embolism severity index [21] and shock index [22]) and the haemodynamic status at the time of pulmonary embolism diagnosis predicted mortality, while the RHT characteristics did not. In the subgroup of patients with intermediate-risk pulmonary embolism (i.e. right ventricular dysfunction), those with RHT had an increased mortality compared with those without RHT. We found that patients with RHT had a worse 30-day outcome when compared with those without RHT. Interestingly, the risk of death among low-risk patients with RHT was about seven-times higher than in patients without RHT. Also, in the subgroup of patients with intermediate-risk pulmonary embolism, those with RHT had a three-fold increased mortality compared with those without RHT. Our study's large sample size, the adjustment for potential confounders and the robustness of the findings provides strong evidence supporting the concept that RHT at the time of acute pulmonary embolism diagnosis is a predictor of all-cause death, pulmonary embolism-related death, and recurrent VTE, particularly in those without haemodynamic compromise.

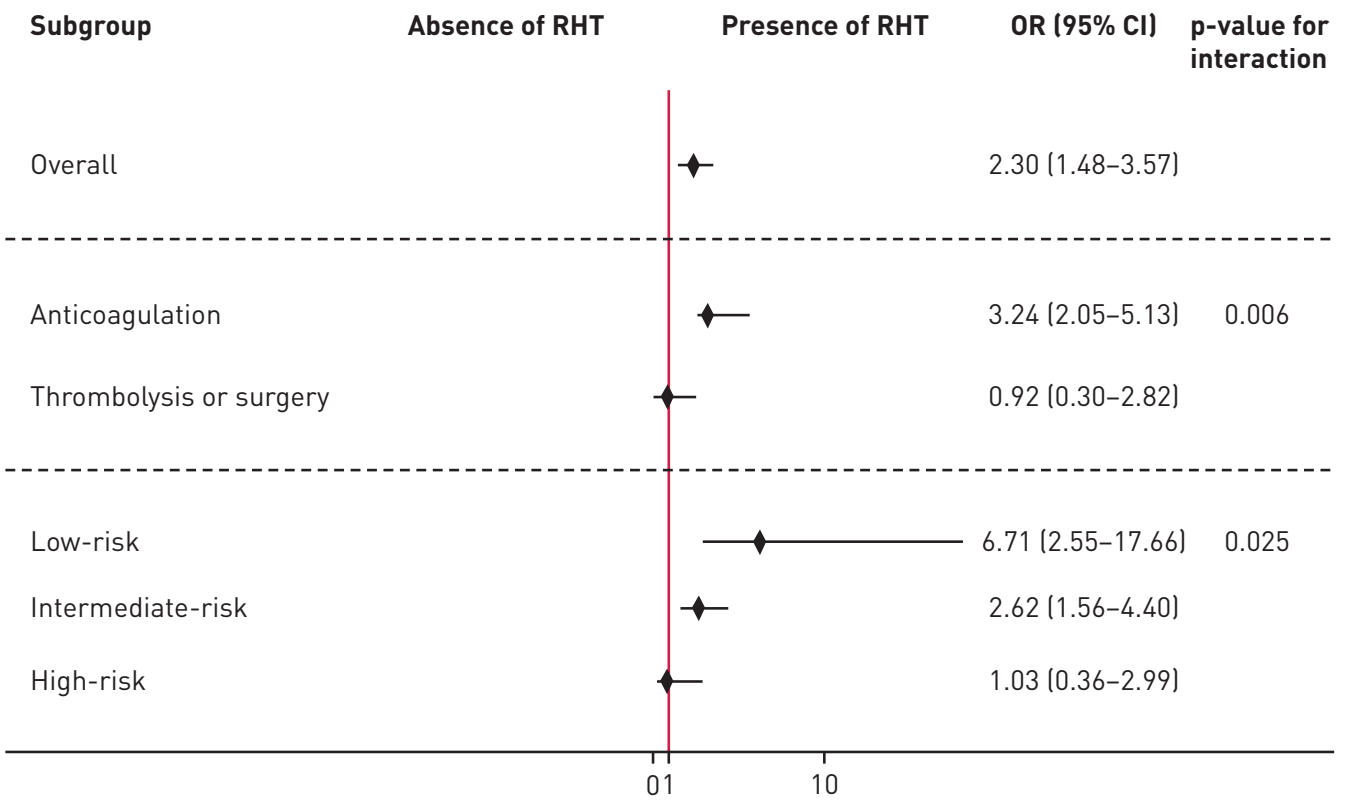

FIGURE 3 Prognostic significance of right heart thrombi (RHT) in the pre-specified subgroups. 
Risk assessment in an individual patient should lead to tailoring of the intensity and type of treatment of acute symptomatic pulmonary embolism [23]. In this study, clinicians used thrombolysis or surgery more frequently in patients who had RHT, and this approach influenced the prognostic significance of clots in the right side of the heart. Among patients who received anticoagulation, the risk of death was about three-times high in those with RHT than in those without RHT. Obviously, changes in treatment, such as the routine use of thrombolysis or surgery, cannot be recommended on the basis of our findings in this cohort analysis.

What are the clinical implications of our findings for the future management of pulmonary embolism? First, physicians should increase their awareness of the potential for RHT in younger patients with pulmonary embolism who present with syncope, hypotension and/or hypoxaemia, particularly if there is a history of recent major bleeding, cancer or congestive heart failure. Second, for patients with RHT, research should focus on improving the initial treatment of pulmonary embolism in order to improve outcomes. Though randomised controlled trials would provide the strongest evidence regarding treatment efficacy and safety for RHT, there might be reasonable concern about excess complexity, expense, and time required to recruit study participants, as well as inadequate representativeness. Thus, well-designed observational studies should be carried out to examine and infer treatment effects.

RIETE is a prospective registry; the data reported are not from a prospective randomised controlled trial. As in most registries, neither diagnostic work-ups nor therapy were controlled. However, the strength of this report is the prospective collection of data from actual practice, from a very large number of pulmonary embolism patients based on diagnostic testing and clinical practices in a wide cross-section of institutions. Another drawback is the lack of clinical detail [24]. Although information on some potential confounders could not be adequately captured (e.g. intensity of anticoagulation), our study design and several sensitivity analyses have attempted to capture and reduce information and selection biases.

In conclusion, the results of this study of patients presenting with acute symptomatic pulmonary embolism suggest that RHT is relatively infrequent. Younger age, heart failure, cancer, recent bleeding, syncope, hypotension and hypoxaemia were associated with an increased risk of RHT. Since patients with concomitant RHT, compared with those without RHT, had worse prognosis, future diagnostic and therapeutic strategies should strive to minimise their consequences.

\section{Acknowledgements}

We express our gratitude to Sanofi Spain for supporting this Registry with an unrestricted educational grant. We also express our gratitude to Bayer Pharma AG for supporting this Registry. Bayer Pharma AG's support was limited to the part of RIETE outside Spain, which accounts for a $22.90 \%$ of the total patients included in the RIETE Registry. We also thank the RIETE Registry Coordinating Center, S \& H Medical Science Service, for their quality control data, logistic and administrative support.

Author contributions are as follows. Study concept and design: D. Barrios, V. Rosa-Salazar, D. Jiménez and M. Monreal; acquisition of data, analysis and interpretation of data, and statistical analysis: D. Barrios, V. Rosa-Salazar, D. Jiménez, R. Morillo, A. Muriel, J. del Toro, L. López-Jiménez, D. Farge-Bancel, R. Yusen and M. Monreal; drafting of the manuscript: D. Barrios, V. Rosa-Salazar, D. Jiménez, R. Morillo, R. Yusen and M. Monreal; critical revision of the manuscript for important intellectual content: D. Barrios, V. Rosa-Salazar, D. Jiménez, R. Morillo, A. Muriel, J. del Toro, L. López-Jiménez, D. Farge-Bancel, R. Yusen and M. Monreal; study supervision: D. Jiménez and M. Monreal. The corresponding author, D. Jiménez, had full access to all the data in the study and had final responsibility for the decision to submit for publication.

The RIETE investigators are as follows. Coordinator of the RIETE Registry: M. Monreal (Spain). RIETE Steering Committee Members: H. Decousus (France), P. Prandoni (Italy), B. Brenner (Israel). RIETE National Coordinators: R. Barba (Spain), P. Di Micco (Italy), L. Bertoletti (France), I. Tzoran (Israel), A. Reis (Portugal), M. Bosevski (Republic of Macedonia), H. Bounameaux (Switzerland), R. Malý (Czech Republic), P. Wells (Canada), M. Papadakis (Greece). RIETE Registry Coordinating Center: S \& H Medical Science Service. Members of the RIETE Group: Spain: M.A. Aibar, M. Alfonso, V. Andújar, J.I. Arcelus, A. Ballaz, R. Barba, M. Barrón, B. Barrón-Andrés, J. Bascuñana, J. Binetti, A. Blanco-Molina, G. Cañada, I. Cañas, I. Casado, J. de Miguel, J. del Toro, M.C. Díaz, S. Díaz, J.A. Díaz-Peromingo, C. Falgá, C. Fernández-Capitán, C. Font, L. Font, P. Gallego, F. García-Bragado, V. Gómez, J. González, E. Grau, L. Guirado, J. Gutiérrez, G. Hernández-Comes, L. Hernández-Blasco, L. Jara-Palomares, M.J. Jaras, D. Jiménez, J.L. Lobo, M. López, L. López-Jiménez, R. López-Reyes, J.B. López-Sáez, M.A. Lorente, A. Lorenzo, O. Madridano, P.J. Marchena, M. Martín, J.M. Martín-Antorán, F. Martín-Martos, M. Modesto, M. Monreal, M.V. Morales, D. Nauffal, J.A. Nieto, S. Nieto, M.J. Núñez, S. Otalora, R. Otero, B. Pagán, J.M. Pedrajas, C. Pérez-Ductor, G. Pérez, I. Pérez, M.L. Peris, I. Pons, J.A. Porras, O. Reig, A. Riera-Mestre, A. Rivas, M.A. Rodríguez-Dávila, V. Rosa, E.M. Rosillo-Hernández, P. Ruiz-Artacho, R. Ruiz-Ferreras, N. Ruiz-Giménez, J.C. Sahuquillo, M.C. Sala-Sainz, A. Sampériz, R. Sánchez, O. Sanz, S. Soler, I. Suárez-Lorenzo, J.M. Suriñach, C. Tolosa, J. Trujillo-Santos, F. Uresandi, B. Valero, R. Valle, A. Vargas, J. Vela, J. Villalta, B. Xifre; Belgium: T. Vanassche, P. Verhamme; Canada: P. Wells; Czech Republic: J. Hirmerova, R. Malý, T. Tomko; Ecuador: E. Salgado; France: L. Bertoletti, A. Bura-Riviere, K. Champion, D. Farge-Bancel, A. Hij, I. Mahé, A. Merah, I. Quere; Greece: M. Papadakis; Israel: A. Braester, B. Brenner, I. Tzoran; Italy: A. Apollonio, G. Barillari, F. Bilora, E. Bucherini, M. Ciammaichella, F. Dentali, P. De Ciantis, P. Di Micco, R. Duce, P. Ferrazzi, E. Grandone, G. Lessiani, C. Lodigiani, D. Mastroiacovo, F. Pace, R. Pesavento, M. Pinelli, R. Poggio, P. Prandoni, M. Rosa, L. Rota, E. Tiraferri, A. Tufano, A. Visonà; Latvia: A. Skride; Portugal: J. Malheiro, J.L. Ribeiro, M.S. Sousa; Republic of Macedonia: M. Bosevski, M. Zdraveska; Switzerland: H. Bounameaux, L. Mazzolai. 


\section{References}

1 Jimenez D, Guijarro R, Trujillo-Santos J, et al. Trends in the management and outcomes of acute pulmonary embolism: analysis from the RIETE registry. J Am Coll Cardiol 2016; 67: 162-170.

2 Laporte S, Mismetti P, Décousus H, et al. Clinical predictors for fatal pulmonary embolism in 15,520 patients with venous thromboembolism: findings from the Registro Informatizado de la Enfermedad TromboEmbolica venosa (RIETE) Registry. Circulation 2008; 117: 1711-1716.

3 Goldhaber SZ, Visani L, De Rosa M. Acute pulmonary embolism: clinical outcomes in the International Cooperative Pulmonary Embolism Registry (ICOPER). Lancet 1999; 353: 1386-1389.

4 Kearon C, Akl EA, Ornelas J, et al. Antithrombotic therapy for VTE disease: CHEST Guideline. Chest 2016; 149: 315-352.

5 Konstantinides SV, Torbicki A, Agnelli G, et al. 2014 ESC Guidelines on the diagnosis and management of acute pulmonary embolism. Eur Heart J 2014; 35: 3033-3073.

6 Chartier L, Bera J, Delomez M, et al. Free-floating thrombi in the right heart: diagnosis, management, and prognostic indexes in 38 consecutive patients. Circulation 1999; 99: 2779-2783.

7 European Working Group on Echocardiography. The European Cooperative Study on the clinical significance of right heart thrombi. Eur Heart J 1989; 10: 1046-1059.

8 Koc M, Kostrubiec M, Elikowski W, et al. Outcome of patients with right heart thrombi: Right Heart Thrombi European Registry. Eur Respir J 2016; 47: 869-875.

9 Lopez-Jimenez L, Montero M, Gonzalez-Fajardo JA, et al. Venous thromboembolism in very elderly patients: findings from a prospective registry (RIETE). Haematologica 2006; 96: 1046-1051.

10 Muriel A, Jiménez D, Aujesky D, et al. Survival effects of inferior vena cava filter in patients with acute symptomatic venous thromboembolism and a significant bleeding risk. J Am Coll Cardiol 2014; 63: 1675-1683.

11 Riera-Mestre A, Jiménez D, Muriel A, et al. Thrombolytic therapy and outcome of patients with an acute symptomatic pulmonary embolism. J Thromb Haemost 2012; 10: 751-759.

12 PIOPED investigators. Value of ventilation/perfusion scan in acute pulmonary embolism: results of the prospective investigation of the pulmonary embolism diagnosis (PIOPED). JAMA 1990; 263: 2753-2759.

13 Remy-Jardin M, Remy J, Wattinne L, et al. Central pulmonary thromboembolism: diagnosis with spiral volumetric CT with the single-breath-hold-technique-comparison with pulmonary angiography. Radiology 1992; 185: 381-387.

14 Kearon C, Ginsberg JS, Hirsh J. The role of venous ultrasonography in the diagnosis of suspected deep venous thrombosis and pulmonary embolism. Ann Intern Med 1998; 129: 1044-1049.

15 Prandoni P, Cogo A, Bernardi E, et al. A simple approach for detection of recurrent proximal vein thrombosis. Circulation 1993; 88: 1730-1735.

16 Nieto JA, Tuesta AD, Marchena PJ, et al. Clinical outcome of patients with venous thromboembolism and recent major bleeding: findings of a prospective registry (RIETE). J Thromb Haemost 2005; 3: 703-709.

17 Kaplan EL, Meier P. Nonparametric estimation from incomplete observations. J Am Stat Assoc 1958; 53: 457-481.

18 Kinney EL, Wright RJ. Efficacy of treatment of patients with echocardiographically detected right-sided heart thrombi: a meta-analysis. Am Heart J 1989; 118: 569-573.

19 Torbicki A, Galie N, Covezzoli A, et al. Right heart thrombi in pulmonary embolism. J Am Coll Cardiol 2003; 41: 2245-2251.

20 Cameron J, Pohlner PG, Stafford EG, et al. Right heart thrombus recognition, diagnosis and management. J Am Coll Cardiol 1985; 5: 1239-1243.

21 Jiménez D, Aujesky D, Moores L, et al. Simplification of the pulmonary embolism severity index for prognostication in patients with acute symptomatic pulmonary embolism. Arch Intern Med 2010; 170 : $1383-1389$.

22 Sam A, Sanchez D, Gomez V, et al. The shock index and the simplified PESI for identification of low-risk patients with acute pulmonary embolism. Eur Respir J 2011; 37: 762-766.

23 Jimenez D, Aujesky D, Yusen RD. Risk stratification of normotensive patients with acute symptomatic pulmonary embolIsm. Br J Haematol 2010; 151: 415-424.

24 Sarrazin MS, Rosenthal GE. Finding pure and simple truths with administrative data. JAMA 2012; 307: $1433-1435$. 